hen-23.tex

\title{
Heavy-baryon chiral perturbation theory approach to thermal neutron capture on ${ }^{3} \mathrm{He}$
}

\author{
Rimantas Lazauskas,, , * Young-Ho Song, ${ }^{2,3,}$, \\ ${ }^{1}$ IPHC, IN2P3-CNRS/Université Louis Pasteur B.P. 28, \\ F-67037 Strasbourg Cedex 2, France \\ ${ }^{2}$ Department of Physics, Duke University, Durham, NCr708, USA \\ ${ }^{3}$ Department of Physics and Astronomy, \\ University of South Carolina, Columbia, SC 29208, USA \\ ${ }^{4}$ Department of Physics and BAERI, \\ Sungkyunkwan University, Suwon 440-746, Korea
}

(Dated: November 11, 2018)

\begin{abstract}
The cross section for radiative thermal neutron capture on ${ }^{3} \mathrm{He}\left({ }^{3} \mathrm{He}+n \rightarrow{ }^{4} \mathrm{He}+\gamma\right.$; known as the hen reaction) is calculated based on heavy-baryon chiral perturbation theory. The relevant M1 operators are derived up to next-to-next-to-next-to-leading order $\left(\mathrm{N}^{3} \mathrm{LO}\right)$. The initial and final nuclear wave functions are obtained from the rigorous Faddeev-Yakubovski equations for five sets of realistic nuclear interactions. Up to $\mathrm{N}^{3} \mathrm{LO}$, the $\mathrm{M} 1$ operators contain two low-energy constants, which appear as the coefficients of non-derivative two-nucleon contact terms. After determining these two constants using the experimental values of the magnetic moments of the triton and ${ }^{3} \mathrm{He}$, we carry out a parameter-free calculation of the hen cross section. The results are in good agreement with the data.
\end{abstract}

PACS numbers: 25.40.Lw,23.20.-g,25.10.+s,11.10.Hi

Keywords:

*Electronic address: rimantas.lazauskas@ires.in2p3.fr

$\dagger$ Electronic address: yhsong@phy.duke.edu, song25@mailbox.sc.edu

${ }^{\ddagger}$ Electronic address: tspark@kias.re.kr 


\section{INTRODUCTION}

The radiative capture of a slow neutron on ${ }^{3} \mathrm{He}\left({ }^{3} \mathrm{He}+n \rightarrow{ }^{4} \mathrm{He}+\gamma\right)$, or the hen process, is an example of rare situations where the contributions of the single-nucleon (1B) currents are strongly suppressed owing to the so-called pseudo-orthogonality, which refers to the fact that the major components of the initial and final states belong to different representations of the

spatial symmetry group and hence cannot be connected by the $r$-independent leading $1 \mathrm{~B}$ operators. To be more specific, the hen reaction proceeds from a $J^{\pi}=1^{+} n+{ }^{3}$ He state whose dominant component belongs to a [31] representation of the spatial permutation group, while the final $\alpha$-particle state belongs to a [4] representation, and these two representations cannot be connected by the leading Gamow-Teller operator, $\vec{\tau} \vec{\sigma}$. This suppression is so drastic that the meson-exchange-current (MEC) "corrections" become comparable to the 1B contributions. Furthermore, it turns out that the MEC and $1 \mathrm{~B}$ terms in this case come with opposite signs, leading to a further drastic suppression of the hen cross section.

The hen process near threshold is governed by the M1 operators (as both the initial and the final states are dominated by the $S$ waves at low energy), and the MEC contributions to them consist of the well-known one-pion-exchange part and the short-range part. Note that the latter is not constrained by the symmetries of QCD, and that, because of the abovementioned suppression and the cancellation mechanisms, the short-range contributions are crucially important even for a rough estimation of the cross section. Furthermore, the strong suppression of the 1B matrix elements (MEs) implies that their values are sensitive to the details of the wave functions. Therefore, for a precise estimation of the hen cross section, it is imperative to have: (i) a reliable method for deriving the relevant MEC operators with a good control of short-range physics, and (ii) the accurate wave functions for the initial and final nuclear states. These requirements make the quantitative estimation of the hen cross section highly nontrivial.

At the same time, the strong enhancement of the relative importance of MEC in the hen process makes it a valuable testing ground for the roles of MEC in light nuclei. Apart from this point, which is important on its own right, a careful study of the hen process is also of great significance in connection with the so-called hep processes, ${ }^{3} \mathrm{He}+p \rightarrow{ }^{4} \mathrm{He}+\nu_{e}+e^{+}$, because hep shares all the aforementioned features of hen: the drastic suppression of the $1 \mathrm{~B}$ contributions, strong cancellation between the $1 \mathrm{~B}$ and the $2 \mathrm{~B}$ contributions, and the 
sensitivity of the transition amplitude to the details of the nuclear wave functions. The hep process is one of the proton-burning reactions that take place in the interior of the sun, and because hep produces the highest-energy solar neutrinos, it has attracted much attention in the study of the solar neutrinos (see Refs. [1, 2] for a recent review) and motivated a series of elaborated studies [3 6]. Park et al. [7] developed an effective field theory (EFT) approach, which has come to be known as "more-effective EFT" (MEEFT for short) [8] and, with the use of MEEFT, they calculated the hep S-factor with an estimated accuracy of about $15 \%$. In view of the fact that the previous theoretical estimations of the hep $S$-factor ranged over two orders of magnitude [7], this is a remarkable feat. A direct test of this theoretical result, however, is not possible because of the unfeasibility of the hep cross section measurement. Meanwhile, the threshold hen cross section has been measured with reasonable accuracy: $\sigma_{\text {exp }}=(54 \pm 6) \mu b[9]$ and $\sigma_{\text {exp }}=(55 \pm 3) \mu b[10]$. Given the close similarity between hen and hep, it is expected that comparison between theory and experiment for the hen case offers valuable information on the validity of the theoretical framework employed for the hep calculation in Ref. [7]. This gives an additional motivation for carrying out a detailed study of hen.

Although the theoretical investigation of hen has a long history, the hen cross section has never been explained in a satisfactory manner. Towner and Khanna [11] and Wervelman et al. [10] performed shell-model calculations for schematic Hamiltonians and obtained $\sigma=$ $(14 \sim 125) \mu b$ and $\sigma=(47 \pm 18) \mu b$, respectively. Much more elaborate calculations with the use of realistic Hamiltonians have been performed by Carlson et al. [14] and by Schiavilla et al. [6], who arrived at $\sigma=112 \mu b$ and $\sigma=86 \mu b$, respectively. These works are based on the conventional approach, the so-called standard nuclear physics approach (SNPA for short), which consists in the use of phenomenological transition operators and phenomenological wave functions. SNPA has been enormously successful in correlating and explaining a vast range of electroweak nuclear transitions in nuclei but, from a formal point of view, it has an insufficiency that it is not equipped with a systematic way of reducing the uncertainty in the MEC operators. The MEC operators in SNPA are constructed based on the approach of Chemtob and Rho [15]. Although this construction of the MEC operators is guided by chiral symmetry and the Ward identities, it is in general unavoidable to have "model-dependent terms".

In this paper we report on a parameter-free MEEFT calculation for the hen cross sec- 
tion at threshold, adopting essentially the same method as used in [7]. ${ }^{\# 1}$ In MEEFT the transition operators are derived from the systematic expansion of the heavy-baryon chiral perturbation theory (HBChPT), and the nuclear MEs are obtained by sandwiching these operators between the wave functions generated from a high-precision phenomenological nuclear potential. Mismatch in the short-range part of the wave function is overcome by the renormalization procedure for the local operators, according to the premise of low-energy EFTs (see below). Thus MEEFT takes advantage of the systematic nature of EFTs and the availability of state-of-the-art wave functions. The mentioned "formal" mismatch may be regarded as a weak point, and also the accurate reproduction of the relevant effective-range parameters (ERPs) is not automatically guaranteed in MEEFT; we come back to these points later.

In the present work we derive the M1 operators within HBChPT up to $\mathrm{N}^{3} \mathrm{LO}$. These M1 operators turn out to contain two low-energy constants (LECs), denoted $g_{4 s}$ and $g_{4 v}$, which are the coefficients of two-nucleon contact-term operators. These LECs can be fixed by requiring that the experimental values of the magnetic moments of the triton and ${ }^{3} \mathrm{He}$, $\mu\left({ }^{3} \mathrm{H}\right)$ and $\mu\left({ }^{3} \mathrm{He}\right)$, be reproduced; this is the same strategy as adopted in Refs. [20, 21], where the M1 properties of the $A=2$ and $A=3$ systems were studied in MEEFT. A remark is in order here on how the short-range contributions are taken into account in MEEFT. The basic premise of EFT is that physics pertaining to ranges shorter than the experimentally relevant scale is to be lodged in local operators. This means that, provided that a proper renormalization procedure is implemented to the coefficients of the local operators (i.e., LECs), the renormalization invariance ensures that the net physical amplitudes be independent of the details of short-range physics. We implement the renormalization condition here by adjusting the values of LECs $\left(g_{4 s}\right.$ and $g_{4 v}$ for hen and $\hat{d}^{R}$ for hep) so as to reproduce a set of known experimental data $\left[\mu\left({ }^{3} \mathrm{H}\right)\right.$ and $\mu\left({ }^{3} \mathrm{He}\right)$ for hen and the tritium-beta-decay rate for hep ]. This matching procedure should be done for each cutoff value and for each potential adopted. Differences in short-range contributions calculated for each case shift the values of LECs (which are not physical observables), but the physical amplitudes should remain unaffected if renormalization invariance is to hold. The validity of this scheme can

\footnotetext{
\#1 There has been an attempt by Song et al. [16] to carry out an MEEFT calculation of hen, but an approximate treatment of the scattering wave function in [16] limits its reliability.
} 
be checked by monitoring the stability of the relevant physical observables with respect to changes in the cutoff parameter $\Lambda$. It turns out (see below) that, in the present hen case, the inclusion of the local-operator (or contact term) contributions reduces the $\Lambda$-dependence by a factor of $\sim 5$, demonstrating the validity of the adopted renormalization procedure. The residual $\Lambda$-dependence may be ascribed to higher order contributions.

One might also worry about the current conservation of MEEFT at short-range. Although the M1 operators arise from the transverse parts of the currents (which by definition have vanishing divergence), current conservation is still relevant in the present context. The reason is that, since most of the Feynman diagrams generate both longitudinal and transverse parts simultaneously, the current conservation breaking in the longitudinal part of the calculated current signals possible mismatch in the M1 operator. This can be part of the aforementioned mismatch problems in MEEFT. The consequences of current conservation breaking were studied by Pastore et al. and Kolling et al. [17-19] and, according to these works, current conservation violation has only minor effects. We have also compared our current operators with those given in these references, which use slightly different power counting schemes. We have found that, after taking into account the renormalization for the LECs and the fact that the contact terms are effective only at $S$-waves, there is no difference in the two-pion and shorter-ranged contributions. In the long-range region, however, there are additional Sachs terms in Ref.[12, 13] whose coefficients are fixed by the nuclear Hamiltonian. \#2 Although the omission of those terms results in a violation of exact current conservation and leads to certain formal mismatches between the current structure and the potential, it is beyond the scope of the present work to solve these problems fully. Here we take the viewpoint that the numerical consequences of these mismatches can be inferred from the cutoff-dependence of the calculated values of observables. It is reassuring that, in our case, this cut-off dependence turns out to be very weak (see Table IV).

In the above we have focused on the short-range contributions. It is however important to note that, for $A$-body systems with $A \geq 3$, even a so-called realistic nuclear interaction often fails to reproduce accurately the ERPs that govern the long-range part of the transition matrix elements. If such a mismatch in the long-range region occurs, it cannot be cured by

\#2 After the submission of our paper, a full EFT calculation of the HEN process became available 12 , 13]. The numerical results in this latest calculation are close to those of our work here, despite differences in the formalisms. 
the renormalization of local operators, a problem that can seriously affect the reliability of a calculated transition amplitude. As an exception to this general statement, however, we should mention that, if a clean correlation between the ERPs and the transition amplitude under consideration can be established, this correlation can be used to drastically reduce the model dependence of the calculated transition amplitude [21]. This point will be explained in more detail later (Sec. III A). Here we simply state that, by taking advantage of this feature, we obtain, as the best estimates for the threshold hen cross section, $\sigma=(49.4 \pm 8.5) \mu b$ (for the AV18+UIX potential), and $(44.4 \pm 6.7) \mu b$ (for the I-N3LO+UIX* potential); see eq.(22) for details. Good agreement of these estimates with the experimental value of the hen cross section gives strong support for the previous MEEFT calculation for the hep S-factor [7].

We wish to emphasize that the present work is the first calculation of hen that employs fully realistic nuclear wave functions $\# 3$; these wave functions are numerically exact solutions to the Faddeev-Yakubovsky equations in configuration space for a specified realistic nuclear interaction. It is to be noted that hitherto even the most advanced realistic calculations [6, 14, 16] disregarded the coupling of the $n^{3}{ }^{3} \mathrm{He}$ to the $p^{3}{ }^{3} \mathrm{H}$ state in the asymptote of the initial wave function. This can have significant numerical consequences in evaluating the $1 \mathrm{~B}$ contributions; see Sec. IIIB, for details.

This paper is organized as follows. In Sec. II we explain the formalisms used to derive the M1 operators and to obtain the four-body nuclear wave functions. Sec. III gives numerical results and analyses. In the final section the implication of our work is discussed.

\section{FORMALISM}

\section{A. Electromagnetic M1 operators and the hen cross section}

In this section we present M1 operators that arise from the multipole expansion of the electromagnetic (EM) currents obtained from HBChPT up to $\mathrm{N}^{3} \mathrm{LO}$ in chiral order counting. HBChPT contains nucleons and pions as pertinent degrees of freedom, with all the other massive fields integrated out. In HBChPT, the EM currents (and consequently the M1 operators) are expanded systematically with increasing powers of $Q / \Lambda_{\chi}$, where $Q$ stands for

\#3 After submission of our manuscript, another EFT calculation of the hen process has appeared[12], where both the potential and current operator are derived in EFT. 
the typical momentum scale of the process and/or the pion mass; $\Lambda_{\chi} \sim 4 \pi f_{\pi} \sim m \sim 1 \mathrm{GeV}$ is the chiral scale, where $f_{\pi} \simeq 92.4 \mathrm{MeV}$ is the pion decay constant, and $m$ is the nucleon mass. We remark that, while the nucleon momentum $\boldsymbol{p}_{i}$ is of the order of $Q$, its energy $\left(\sim \boldsymbol{p}_{i}^{2} / m\right)$ is of the order of $Q^{2} / m$, and consequently the four-momentum of the emitted photon $q^{\mu}=(\omega, \boldsymbol{q})$ should also be counted as $\mathcal{O}\left(Q^{2} / m\right)$.

We derive the MEC operators from the non-relativistic reduction of irreducible contributions of Feynman diagrams in HBChPT. Irreducible contributions coming from box diagrams are obtained by removing pure nucleon-pole contributions. As mentioned, it is to be noted that there exist other approaches to deriving MEC operators from HBChPT [17-19]. Although detailed comparison of our formalism with these approaches should be informative, we relegate it to future studies.

The M1 operator $\boldsymbol{\mu}_{1 M}(q)$ is defined as

$$
\boldsymbol{\mu}_{1 M}(q) \equiv\left(\frac{i q}{\sqrt{6 \pi}}\right)^{-1} \hat{T}_{1 M}^{M a g}(q)
$$

with

$$
\hat{T}_{J M}^{M a g}(q) \equiv \int d^{3} \boldsymbol{x}\left[j_{J}(q x) \boldsymbol{Y}_{J J 1}^{M}(\hat{\boldsymbol{x}})\right] \cdot \boldsymbol{j}(\boldsymbol{x}),
$$

where $q \equiv|\boldsymbol{q}|=20.578 \mathrm{MeV}, j_{J}(q x)$ is the spherical Bessel function of order $J, \boldsymbol{Y}_{J J 1}^{M}(\hat{\boldsymbol{x}})$ is the vector spherical harmonics, and $\boldsymbol{j}(\boldsymbol{x})$ is the EM current operator. We have chosen the normalization of $\boldsymbol{\mu}_{1 M}(q)$ such that it becomes the usual magnetic dipole moment in zero $q$ limit.

In terms of $\boldsymbol{\mu}_{1 M}(q)$, the hen cross section at thermal energy is given by

$$
\sigma=\alpha \pi \frac{c}{v_{r e l}}\left(\frac{q}{m c^{2}}\right)^{2}\left(\frac{q}{\hbar c}\right)|\mathcal{M}|^{2}
$$

with

$$
\mathcal{M} \equiv\left\langle\Psi_{4 \mathrm{He}}^{J=0, M=0}\left|\boldsymbol{\mu}_{11}(q)\right| \Psi_{n^{3} \mathrm{He}}^{J=1, M=-1}\right\rangle
$$

where $\alpha$ is the fine structure constant, $m$ is the nucleon mass and $v_{r e l}=2200 \mathrm{~m} / \mathrm{s}$ is the thermal neutron velocity.

The detailed full forms of the M1 operators up to $\mathrm{N}^{3} \mathrm{LO}$ are given in our recent papers [20, 21], which we briefly summarize here. The M1 operators up to $\mathrm{N}^{3} \mathrm{LO}$ consist of one-body(1B) and two-body $(2 \mathrm{~B})$ contributions; three-body operators enter only at $\mathrm{N}^{4} \mathrm{LO}$ or higher orders in our counting scheme. 
The 1B M1 operators read, in the center of mass frame,

$$
\begin{aligned}
\boldsymbol{\mu}_{1 \mathrm{~B}}(q)= & \frac{1}{2 m} \sum_{i}\left\{\hat{j}_{0}\left(q r_{i}\right)\left[\boldsymbol{\sigma}_{i}\left(\mu_{i}-Q_{i} \frac{\overline{\boldsymbol{p}}_{i}^{2}}{2 m^{2}}\right)-\frac{\mu_{i}-Q_{i}}{2 m^{2}} \overline{\boldsymbol{p}}_{i} \boldsymbol{\sigma}_{i} \cdot \overline{\boldsymbol{p}}_{i}\right]\right. \\
& +\hat{j}_{1}\left(q r_{i}\right)\left[Q_{i} \boldsymbol{r}_{i} \times \overline{\boldsymbol{p}}_{i}\left(1-\frac{\overline{\boldsymbol{p}}_{i}^{2}}{2 m^{2}}\right)-\frac{w\left(2 \mu_{i}-Q_{i}\right)}{4 m} i \boldsymbol{r}_{i} \times\left(\overline{\boldsymbol{p}}_{i} \times \boldsymbol{\sigma}_{i}\right)\right] \\
& \left.+\frac{\left(q r_{i}\right)^{2}}{30} \hat{j}_{2}\left(q r_{i}\right) \mu_{i}\left(3 \hat{\boldsymbol{r}}_{i} \hat{\boldsymbol{r}}_{i} \cdot \boldsymbol{\sigma}_{i}-\boldsymbol{\sigma}_{i}\right)+\cdots\right\},
\end{aligned}
$$

where $\hat{j}_{n}(x) \equiv \frac{(2 n+1) ! !}{x^{n}} j_{n}(x), Q_{i}$ and $\mu_{i}$ are the charge and magnetic moments of the $i$-th nucleon, respectively, and $\overline{\boldsymbol{p}}_{i} \equiv \frac{1}{2}\left(i \bar{\nabla}_{i}-i \vec{\nabla}_{i}\right)$ is the mean momentum operator of the $i$-th nucleon.

The two-body M1 operators \#4 up to $\mathrm{N}^{3} \mathrm{LO}$ can be divided into four types: the soft-onepion-exchange $(1 \pi)$ term, the vertex correction to the one-pion exchange $(1 \pi C)$ term, the two-pion-exchange $(2 \pi)$ term, and the contact-term contribution $(\mathrm{CT})$,

$$
\boldsymbol{\mu}_{2 \mathrm{~B}}(q)=\sum_{i<j}\left[\boldsymbol{\mu}_{i j}^{1 \pi}+\left(\boldsymbol{\mu}_{i j}^{1 \pi C}+\boldsymbol{\mu}_{i j}^{2 \pi}+\boldsymbol{\mu}_{i j}^{\mathrm{CT}}\right)\right]=\mathrm{NLO}+\mathrm{N}^{3} \mathrm{LO}
$$

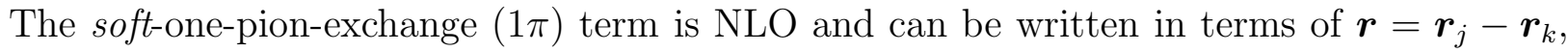
$r=|\boldsymbol{r}|, \hat{\boldsymbol{r}}=\boldsymbol{r} /|\boldsymbol{r}|, \boldsymbol{R}=\left(\boldsymbol{r}_{j}+\boldsymbol{r}_{k}\right) / 2, R=|\boldsymbol{R}|, S_{j k}=3 \boldsymbol{\sigma}_{j} \cdot \hat{\boldsymbol{r}} \boldsymbol{\sigma}_{k} \cdot \hat{\boldsymbol{r}}-\boldsymbol{\sigma}_{j} \cdot \boldsymbol{\sigma}_{k}, g_{A} \simeq 1.2695$,

$$
\begin{aligned}
\boldsymbol{\mu}_{j k}^{1 \pi} & =\frac{g_{A}^{2}}{8 f_{\pi}^{2}}\left[\hat{T}_{S}^{(\times)}\left(\frac{2}{3} y_{1 \Lambda}^{\pi}(r)-y_{0 \Lambda}^{\pi}(r)\right)-\hat{T}_{T}^{(\times)} y_{1 \Lambda}^{\pi}(r)\right] \hat{j}_{0}(q R) \\
& -\frac{g_{A}^{2} m_{\pi}^{2}}{24 f_{\pi}^{2}} \tau_{\times}^{z} \boldsymbol{R} \times \boldsymbol{r}\left[\boldsymbol{\sigma}_{1} \cdot \boldsymbol{\sigma}_{2} \bar{y}_{0 \Lambda}^{\pi}(r)+S_{j k} y_{2 \Lambda}^{\pi}(r)\right] \hat{j}_{1}(q R)+\cdots
\end{aligned}
$$

where, $\hat{T}_{S}^{(\odot)} \equiv \tau_{\odot}^{z} \boldsymbol{\sigma}_{\odot}$ and $\hat{T}_{T}^{(\odot)} \equiv \tau_{\odot}^{z}\left[\hat{\boldsymbol{r}} \hat{\boldsymbol{r}} \cdot \boldsymbol{\sigma}_{\odot}-\frac{1}{3} \boldsymbol{\sigma}_{\odot}\right], \tau_{\odot}=\tau_{1} \odot \tau_{2}, \boldsymbol{\sigma}_{\odot}=\boldsymbol{\sigma}_{1} \odot \boldsymbol{\sigma}_{2}$, with $\odot= \pm, \times$. The cutoff dependence of $2 \mathrm{~B}$ operators is taken into account by introducing a Gaussian regulator with a cutoff $\Lambda$ when performing Fourier transformation of the 2B operators into coordinate space; this procedure gives the regularized delta and Yukawa functions,

$$
\begin{aligned}
\delta_{\Lambda}(r) & \equiv \int \frac{d^{3} \boldsymbol{k}}{(2 \pi)^{3}} e^{-\boldsymbol{k}^{2} / \Lambda^{2}} e^{i \boldsymbol{k} \cdot \boldsymbol{r}}, \\
y_{0 \Lambda}^{\pi}(r) & \equiv \int \frac{d^{3} \boldsymbol{k}}{(2 \pi)^{3}} e^{-\boldsymbol{k}^{2} / \Lambda^{2}} e^{i \boldsymbol{k} \cdot \boldsymbol{r}} \frac{1}{\boldsymbol{k}^{2}+m_{\pi}^{2}}, \\
y_{1 \Lambda}^{\pi} & \equiv-r \frac{d}{d r} y_{0 \Lambda}^{\pi}, \quad y_{2 \Lambda}^{\pi} \equiv \frac{r}{m_{\pi}^{2}} \frac{d}{d r} \frac{1}{r} \frac{d}{d r} y_{0 \Lambda},
\end{aligned}
$$

\#4 In this work we neglect the so-called fixed-current contribution, which is proved to be numerically negligible [20]. 
where, $m_{\pi}$ is the pion mass.

The one-loop vertex correction to one-pion exchange ( $1 \pi C$ term) reads

$$
\begin{aligned}
\boldsymbol{\mu}_{j k}^{1 \pi C}= & -\frac{g_{A}^{2}}{8 f_{\pi}^{2}}\left(\bar{c}_{\omega}+\bar{c}_{\Delta}\right)\left[\left(\hat{T}_{S}^{(+)}+\hat{T}_{S}^{(-)}\right) \frac{\bar{y}_{0 \Lambda}^{\pi}}{3}+\left(\hat{T}_{T}^{(+)}+\hat{T}_{T}^{(-)}\right) y_{2 \Lambda}^{\pi}\right] \hat{j}_{1}(q R) \\
& +\frac{g_{A}^{2}}{8 f_{\pi}^{2}} \bar{c}_{\Delta}\left[\frac{1}{3} \hat{T}_{S}^{(\times)} \bar{y}_{0 \Lambda}^{\pi}-\frac{1}{2} \hat{T}_{T}^{(\times)} y_{2 \Lambda}^{\pi}\right] \hat{j}_{1}(q R) \\
& -\frac{1}{16 f_{\pi}^{2}} \bar{N}_{W Z} \tau_{j} \cdot \tau_{k}\left[\boldsymbol{\sigma}_{+} \bar{y}_{0 \Lambda}^{\pi}+\left(3 \hat{\boldsymbol{r}} \hat{\boldsymbol{r}} \cdot \boldsymbol{\sigma}_{+}-\boldsymbol{\sigma}_{+}\right) y_{2 \Lambda}^{\pi}\right] \hat{j}_{1}(q R),
\end{aligned}
$$

where the values of the LECs, $\left(\bar{c}_{\omega}, \bar{c}_{\Delta}, \bar{N}_{W Z}\right) \simeq(0.1021,0.1667,0.02395)$, are determined from the resonance saturation model[22, 23].

The two-pion exchange $(2 \pi)$ term reads

$$
\begin{aligned}
\boldsymbol{\mu}_{j k}^{2 \pi} & =\frac{1}{128 \pi^{2} f_{\pi}^{4}}\left[\left(\hat{T}_{S}^{(+)}-\hat{T}_{S}^{(-)}\right) L_{S}(r)+\left(\hat{T}_{T}^{(+)}-\hat{T}_{T}^{(-)}\right) L_{T}(r)\right] \hat{j}_{1}(q R) \\
& -\frac{1}{256 \pi^{2} f_{\pi}^{4}}\left(\tau_{j} \times \tau_{k}\right)^{z} \boldsymbol{R} \times \hat{\boldsymbol{r}} \frac{d}{d r} L_{0}(r) \hat{j}_{0}(q R),
\end{aligned}
$$

where

$$
\begin{aligned}
L_{S}(r)= & -\frac{g_{A}^{2}}{3} r \frac{d}{d r} K_{0}+\frac{g_{A}^{4}}{3}\left[4 K_{1}-2 K_{0}+r \frac{d}{d r}\left(K_{0}+2 K_{1}\right)\right], \\
L_{T}(r)= & \frac{g_{A}^{2}}{2} r \frac{d}{d r} K_{0}+\frac{g_{A}^{4}}{2}\left[4 K_{\mathrm{T}}-r \frac{d}{d r}\left(K_{0}+2 K_{1}\right)\right] \\
L_{0}(r)= & 2 K_{2}+g_{A}^{2}\left(8 K_{2}+2 K_{1}+2 K_{0}\right) \\
& \quad-g_{A}^{4}\left(16 K_{2}+5 K_{1}+5 K_{0}\right)+g_{A}^{4} \frac{d}{d r}\left(r K_{1}\right) .
\end{aligned}
$$

The loop functions $K^{\prime} s$ are defined in Ref. [7, 22] .

Note that the contact-term $\boldsymbol{\mu}_{i j}^{\mathrm{CT}}$ contains two low-energy constants (LECs), $g_{4 s}$ and $g_{4 v}$ :

$$
\boldsymbol{\mu}_{i j}^{\mathrm{CT}}=\frac{1}{2 m}\left[g_{4 s}\left(\boldsymbol{\sigma}_{i}+\boldsymbol{\sigma}_{j}\right)+g_{4 v}\left(\vec{\tau}_{i} \times \vec{\tau}_{j}\right)^{z}\left(\boldsymbol{\sigma}_{i} \times \boldsymbol{\sigma}_{j}\right)\right] \delta_{\Lambda}^{(3)}\left(\boldsymbol{r}_{i j}\right) .
$$

Both $g_{4 s}$ and $g_{4 v}$ have the dimension of $\mathrm{fm}^{3}$. Since the values of these LECs are not determined by symmetry arguments, they need to be fixed either by solving QCD at low-energy or by fitting to a set of experimental observables that are sensitive to them. Since the former is not feasible at present, we resort to the latter. Specifically, we fix $g_{4 s}$ and $g_{4 v}$ so as to reproduce the experimental values of $\mu\left({ }^{3} \mathrm{H}\right)$ and $\mu\left({ }^{3} \mathrm{He}\right)$, for each nuclear interaction model adopted and for each cutoff value. 


\section{B. Faddeev-Yakubovsky equations}

The relevant four-nucleon wave functions are obtained by solving the FaddeevYakubovsky (FY) equations in configuration space [24, 25]. The FY formalism offers a mathematically rigorous description for both continuum and bound states. In this formalism wave functions are naturally decomposed into so-called FY amplitudes (FYAs). For $A=4$ systems, there appear two types of FYAs, which we refer to as components $K_{i j, k}^{l}$ and $H_{i j}^{k l}$ where $i, j, k, l$ are particle indices. The asymptotes of the components $K_{i j, k}^{l}$ and $H_{i j}^{k l}$ incorporate $3+1$ and $2+2$ particle channels, respectively (see Fig,1). The FYAs are evalu-
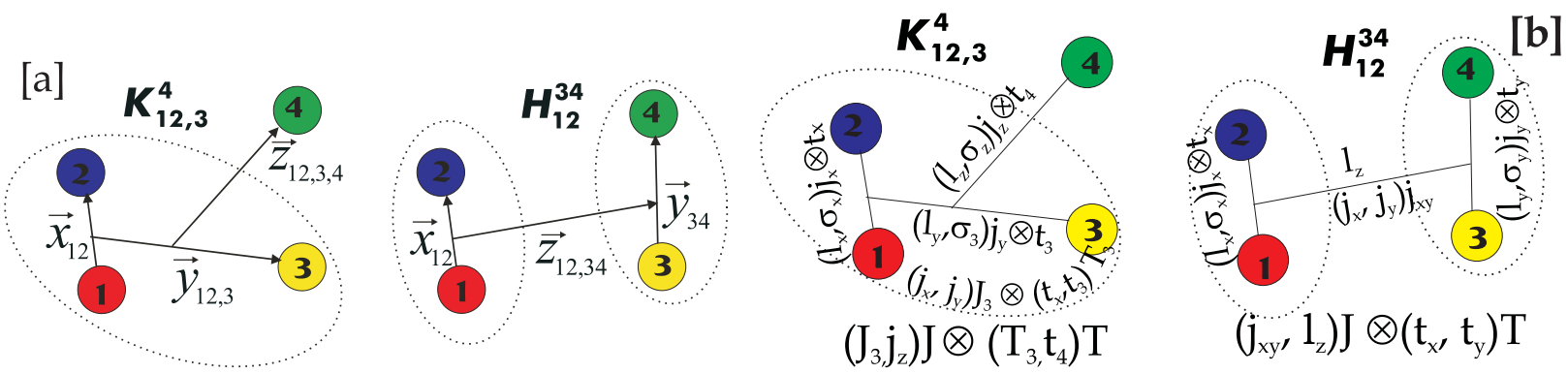

FIG. 1: (Color online) The FY components $K_{12,3}^{4}$ and $H_{12}^{34}$ for a given particle ordering. As $z \rightarrow \infty$, the $K$ components describe $3+1$ particle channels, while the $H$ components contain asymptotic states of $2+2$ channels, see figure [a]. Figure [b] shows the $j$ - $j$ coupling scheme used in expanding $K$ and $H$ into partial wave bases.

ated in the isospin formalism, i.e., protons and neutrons are regarded as degenerate states with the same mass, which is fixed to $\hbar^{2} / m=41.47 \mathrm{MeV} \cdot \mathrm{fm}^{2}$. Three-body forces typically arise from integrating out the higher-energy degrees of freedom, and therefore they can be decomposed as $V_{123}=V_{12}^{3}+V_{23}^{1}+V_{31}^{2}$, where $k$ in $V_{i j}^{k}$ is the particle in a high-energy intermediate state.[31]. In the presence of a three-body force, the FY equations for $K \equiv K_{12,3}^{4}$ and $H \equiv H_{12}^{34}$ read [26, 27]

$$
\begin{aligned}
& \left(E-H_{0}-V_{12}-\sum_{i<j} V_{i j}^{C}\right) K=V_{12}\left(P^{+}+P^{-}\right)[(1+Q) K+H]+\frac{1}{2}\left(V_{23}^{1}+V_{31}^{2}\right) \Psi, \\
& \left(E-H_{0}-V_{12}-\sum_{i<j} V_{i j}^{C}\right) H=V_{12} \tilde{P}[(1+Q) K+H],
\end{aligned}
$$

where $V_{i j}$ and $V_{i j}^{C}$ are, respectively, the short-ranged part and the Coulomb-dominated longrange part of the interaction between the $i$-th and $j$-th nucleons. $P^{+}=\left(P^{-}\right)^{-1} \equiv P_{23} P_{12}$, 
$Q \equiv-P_{34}$ and $\tilde{P} \equiv P_{13} P_{24}=P_{24} P_{13}$, where $P_{i j}$ is the particle permutation operator. In terms of the FYAs, the total wave function of an $A=4$ system is given by

$$
\Psi=\left[1+\left(1+P^{+}+P^{-}\right) Q\right]\left(1+P^{+}+P^{-}\right) K+\left(1+P^{+}+P^{-}\right)(1+\tilde{P}) H .
$$

We expand $K$ and $H$ in terms of the tripolar harmonics $Y_{i}^{\alpha}\left(\hat{x}_{i}, \hat{y}_{i}, \hat{z}_{i}\right)$, which comprise the spins and isospins of the nucleons as well as the angular variables,

$$
\Phi_{i}\left(\vec{x}_{i}, \vec{y}_{i}, \vec{z}_{i}\right)=\sum_{\alpha} \frac{\mathcal{F}_{i}^{\alpha}\left(x_{i}, y_{i}, z_{i}\right)}{x_{i} y_{i} z_{i}} Y_{i}^{\alpha}\left(\hat{x}_{i}, \hat{y}_{i}, \hat{z}_{i}\right),
$$

where $\Phi$ stands for either $K$ or $H$, and the subscript $i$ denotes the particle-grouping class (among the four nucleons). We note that the total angular momentum and its projection, parity and the third component of the isospin $\left(\mathcal{T}_{z}=0\right)$ are good quantum numbers, and the subscript $\alpha$ denotes collectively eleven other non-fixed quantum numbers. We use the $j$-j scheme for the coupling of angular momenta, as illustrated in Fig. 1b. The Jacobi coordinates used here are depicted in Fig. 1. This choice of coordinates allows us to separate the centerof-mass motion and guarantees that the kinetic energy operator is independent of the angular variables.

The expansion of Eq. (13) in terms of the natural configuration space basis leads to coupled integro-differential equations for the radial parts of FYAs $\left(\mathcal{F}_{i}^{\alpha}\left(x_{i}, y_{i}, z_{i}\right)\right)$. Note that, contrary to the ordinary $3 N$ problems, the number of radial parts of FYAs is infinite even when the pair interaction is restricted to a finite number of partial waves. This situation arises from the existence of the additional degree of freedom $l_{z}$ in the expansion of the $K$ type components. In numerical calculations, therefore, we need to introduce an additional truncation by identifying relevant amplitudes and discarding the remainder (see below).

\section{Boundary conditions}

Eqs. (13) needs to be supplemented with appropriate boundary conditions, which can be written in the Dirichlet form. For both bound and scattering states, the radial FYAs satisfy the regularity conditions:

$$
\mathcal{F}_{i}^{\alpha}\left(0, y_{i}, z_{i}\right)=\mathcal{F}_{i}^{\alpha}\left(x_{i}, 0, z_{i}\right)=\mathcal{F}_{i}^{\alpha}\left(x_{i}, y_{i}, 0\right)=0 .
$$

For bound state problems, since the wave functions are compact, the regularity conditions

can be implemented by requiring $\mathcal{F}_{i}^{\alpha}$ to vanish at the borders of the hypercube, $\left[0, X_{\max }\right] \times$ 


$$
\begin{aligned}
& {\left[0, Y_{\max }\right] \times\left[0, Z_{\max }\right],{ }^{\# 5}} \\
& \mathcal{F}_{i}^{\alpha}\left(X_{\max }, y_{i}, z_{i}\right)=\mathcal{F}_{i}^{\alpha}\left(x_{i}, Y_{\max }, z_{i}\right)=\mathcal{F}_{i}^{\alpha}\left(x_{i}, y_{i}, Z_{\max }\right)=0 .
\end{aligned}
$$

The hypercube is chosen large enough to accommodate the wave functions.

On the other hand, a scattering state near threshold contains two coupled channels, $n$ ${ }^{3} \mathrm{He}$ and $p_{-}{ }^{3} \mathrm{H}$, both of which are of type $K$. In this case we impose the following matching condition at $z_{i}=Z_{\max }$ :

$$
\begin{aligned}
\mathcal{K}_{i}^{\alpha}\left(x_{i}, y_{i}, Z_{\max }\right)= & \left.\frac{1}{\sqrt{4}} \sum_{j_{z}^{\prime} l_{z}^{\prime} T_{3}^{\prime z}}\left\{f_{i}^{\alpha_{a}}\left(\boldsymbol{x}_{i}, \boldsymbol{y}_{i}\right)\right\}_{J_{3} \equiv \frac{1}{2}, T_{3} T_{3}^{z}} \otimes\left\{Y_{l_{z}^{\prime}}\left(\widehat{z}_{i}\right) \otimes s_{i}\right\}_{j_{z}^{\prime}}\right\rangle_{J M} \\
\times & \left(\frac{i}{2}\left[\delta_{l_{z}, 0} h_{l_{z}}^{-}\left(p_{n} Z_{\max }\right)-S_{j_{z}^{\prime} l_{z}^{\prime} T_{3}^{\prime z}, j_{z} l_{z} T_{3}^{z}} h_{l_{z}^{\prime}}^{+}\left(p_{n} Z_{\max }\right)\right] C_{T_{3} T_{3}^{\prime z}, \frac{1}{2}-\frac{1}{2}}^{T 0}\right. \\
& \left.-\frac{i}{2} \sqrt{\frac{p_{p}^{\prime}}{p_{n}}} S_{j_{z}^{\prime} l_{z}^{\prime} T_{3}^{\prime z}, j_{z} l_{z} T_{3}^{z}} e^{2 i \sigma_{l_{z}^{\prime}}} u_{l_{z}^{\prime}}^{+}\left(\eta, p_{p}^{\prime} Z_{\max }\right) C_{T_{3} T_{3}^{\prime z}, \frac{1}{2} \frac{1}{2}}^{T 0}\right) .
\end{aligned}
$$

Here $p_{n}=\frac{3}{4 \hbar} m v_{r e l}$ is the neutron momentum in the $n{ }^{3} \mathrm{He}$ channel, while $p_{p}=$ $\left[p_{n}^{2}+\frac{3 m}{2 \hbar^{2}}\left(B_{3^{3} \mathrm{H}}-B_{3^{3} \mathrm{He}}\right)\right]^{1 / 2}$ is the proton momentum in the $p^{-}{ }^{3} \mathrm{H}$ channel; $h_{l_{z}}^{ \pm}$are the spherical Hankel functions, and $u_{l_{z}^{\prime}}^{+}$is the outgoing Coulomb function for the $p-{ }^{3} \mathrm{H}$ channel with $\eta=\frac{4}{3} \alpha m c /\left(\hbar p_{p}\right)$. The functions $\left\{f_{i}^{\alpha_{a}}\left(\boldsymbol{x}_{i}, \boldsymbol{y}_{i}\right)\right\}_{J_{3} \equiv \frac{1}{2}, T_{3} T_{3}^{z}}$ with $T_{3}^{z}= \pm \frac{1}{2}$ are the normalized Faddeev amplitudes for ${ }^{3} \mathrm{He}$ and ${ }^{3} \mathrm{H}$, which we obtain by solving the corresponding $3 \mathrm{~N}$ bound state problems.

We neglect the $\mathcal{T} \neq 0$ components in our ${ }^{4} \mathrm{He}$ bound-state calculation as they represent less than $0.01 \%$ [48] of the total wave function. However, for the rigorous solution of the scattering problem, we cannot use the $\mathcal{T}=0$ approximation; we do need to consider admixtures of $\mathcal{T}=1$ and $\mathcal{T}=2$ states. These admixtures are needed in order to correctly separate the asymptotes of the $n^{-3} \mathrm{He}$ and $p-{ }^{3} \mathrm{H}$ channels, which have different thresholds due to the difference in the ${ }^{3} \mathrm{He}$ and ${ }^{3} \mathrm{H}$ binding energies.

\section{RESULTS}

In this work we have performed rigorous FY calculations for five sets of nuclear potentials: AV18, I-N3LO, INOY, AV18+UIX and I-N3LO+UIX*. Here AV18 stands for the

$\# 5\left(X_{\max }, Y_{\max }, Z_{\max }\right)$ are chosen to be $\left(L_{y}, \sqrt{3 / 4} L_{y}, \sqrt{2 / 3} L_{z}\right)$ for the component $\mathrm{K}$ and $\left(L_{y}, L_{y}, \sqrt{1 / 2} L_{z}\right)$ for the component $\mathrm{H}$, where $L_{y}=25 \mathrm{fm}$ and $L_{z}=(27 \sim 30) \mathrm{fm}$. We have verified that a hypercube size larger than these values does not cause any noticeable changes. 
TABLE I: The hen cross section, $\sigma$, calculated for the five realistic nuclear interactions mentioned in the text. The uncertainties attached to $\sigma$ represent the variation of $\sigma$ as the cutoff parameter $\Lambda$ is varied in the range $\Lambda=(500 \sim 900) \mathrm{MeV}$. Also shown are the calculated values of the binding energies (BE; in units of $\mathrm{MeV}$ ) for ${ }^{3} \mathrm{H},{ }^{3} \mathrm{He}$ and ${ }^{4} \mathrm{He}$, the point-proton rms radius [33] $r_{\mathrm{He} 4}$ (in $\mathrm{fm}$ ), the $D$-state probability $P_{D}\left({ }^{4} \mathrm{He}\right)$ (in per cent) of the $\alpha$-particle, and the spin-triplet $n_{-}{ }^{3} \mathrm{He}$ scattering length, $a_{n \mathrm{He} 3}$ (in units of $\mathrm{fm}$ ).

\begin{tabular}{|c|c|c|c|c|c|c|c|}
\hline & $\mathrm{BE}\left({ }^{3} \mathrm{H}\right)$ & $\mathrm{BE}\left({ }^{3} \mathrm{He}\right)$ & $\mathrm{BE}\left({ }^{4} \mathrm{He}\right)$ & $r_{\mathrm{He} 4}$ & $P_{D}\left({ }^{4} \mathrm{He}\right)$ & $a_{n \mathrm{He} 3}$ & $\sigma[\mu b]$ \\
\hline AV18 & 7.623 & 6.925 & 24.23 & 1.516 & 13.8 & $3.43-0.0082 i$ & $80.0 \pm 12.2$ \\
\hline I-N3LO & 7.852 & 7.159 & 25.36 & 1.52 & 9.30 & $3.56-0.0070 i$ & $57.3 \pm 7.9$ \\
\hline INOY & 8.483 & 7.720 & 29.08 & 1.377 & 5.95 & $3.26-0.0058 i$ & $34.4 \pm 4.5$ \\
\hline AV18+UIX & 8.483 & 7.753 & 28.47 & 1.431 & 16.0 & $3.23-0.0054 i$ & $49.4 \pm 8.5$ \\
\hline $\mathrm{I}-\mathrm{N} 3 \mathrm{LO}+\mathrm{UIX}^{*}$ & 8.482 & 7.737 & 28.12 & 1.475 & 10.9 & $3.44-0.0055 i$ & $44.4 \pm 6.7$ \\
\hline Exp.: & 8.482 & 7.718 & 28.30 & $1.475(6)$ & & \multicolumn{2}{|c|}{$3.278(53)-0.001(2) i 55 \pm 3,54 \pm 6$} \\
\hline
\end{tabular}

Argonne v18 potential [28], I-N3LO for the chiral N32O potential of the Idaho group [29], and INOY for the non-local configuration space potential that has recently been derived by Doleschall [30]; UIX is the tri-nucleon interaction derived by the Urbana group [31]. For the case of I-N3LO+UIX ${ }^{*}$, we have attached an asterisk to indicate that the $A_{2 \pi}$ parameter in UIX has been slightly modified (from -0.0293 to -0.03827 ) so as to reproduce the triton binding energy precisely, see Ref. [21] for details.

For each of these five nuclear interactions, and for each of the three choices of the cutoff parameter, $\Lambda=500,700$ and $900 \mathrm{MeV}$, we determine the LECs, $g_{4 s}$ and $g_{4 v}$, in such a manner that the experimental values of the triton and ${ }^{3} \mathrm{He}$ magnetic moments are reproduced. We then proceed to calculate the hen cross section, $\sigma$. The results are given in Table I. The table also shows the calculated values of the binding energies of ${ }^{3} \mathrm{H},{ }^{3} \mathrm{He}$ and ${ }^{4} \mathrm{He}$, the pointproton rms radius $r_{\mathrm{He} 4}$ of ${ }^{4} \mathrm{He},{ }^{\# 6}$ the $D$-state probability of ${ }^{4} \mathrm{He}$, and the spin-triplet $n-{ }^{3} \mathrm{He}$

\#6 The point-proton rms radius $r_{\mathrm{He} 4}$ is defined as $\left(r_{\mathrm{He} 4}\right)^{2} \equiv r_{c}^{2}\left({ }^{4} \mathrm{He}\right)-r_{p}^{2}-r_{n}^{2}$, where $r_{c}\left({ }^{4} \mathrm{He}\right)$ is the proton charge rms radius of ${ }^{4} \mathrm{He}, r_{p}$ and $r_{n}$ are the rms charge radius of the proton and neutron, respectively. See, for example, 32] for detailed explanation. With the ${ }^{4} \mathrm{He}$ proton charge radius $r_{c}\left({ }^{4} \mathrm{He}\right)=1.681(4) \mathrm{fm}$ obtained in a recent analysis [33] and with the 2008 PDG values for the proton and neutron rms radii, $r_{p}=0.875(7) \mathrm{fm}$ and $r_{n}^{2}=-0.1161(22) \mathrm{fm}^{2}$, we arrive at $r_{\mathrm{He} 4}=1.475(6) \mathrm{fm}$, which is about $1.4 \%$ larger than the estimate given in 32 . 
scattering length, $a_{n \mathrm{He} 3}$.

The bound state properties calculated in this work agree well with those obtained in other calculations [34-36]. Theoretical calculations for $a_{n \mathrm{He}}$ are much less established, but we have checked that our results agree within $2 \%$ with the momentum-space FY calculation of the Lisboa group [37], as well as with the RGM calculation carried out by Hofmann [38] for the AV18 and AV18+UIX potentials.

The table also indicates that the three-nucleon interactions (TNIs) play an important role in bringing the binding energies and $a_{n \mathrm{He} 3}$ close to their respective experimental values. We remark that there is some uncertainty in the experimental value of $a_{n \mathrm{He} 3}$. The value listed in the table is due to an $R$-matrix analysis [39] of the $n-{ }^{3} H e$ scattering data measured before the year 2002. Recently new measurements of the coherent scattering length have been performed at NIST [40] and ILL [41], but the results of the two groups do not agree with each other, and both of them are in disagreement with the old ILL measurement.

The $D$-state probability of the $\alpha$-particles, $P_{D}\left({ }^{4} \mathrm{He}\right)$, which is closely related to the tensor forces, shows strong model dependence. However this quantity is not an observable, and it turns out to be difficult to constrain this quantity by studying other processes that are sensitive to $P_{D}\left({ }^{4} \mathrm{He}\right)[42]$.

As mentioned, to solve the FY equation numerically, we need to introduce truncations in the angular momentum expansion of the FYAs. We implement these truncations by assuming that the partial FYAs with $j_{x}, j_{y}, j_{z}$ (and $l_{z}$ for the type $\mathrm{H}$ ) larger than a specified value, $\max \left(j_{i}\right)$, can be ignored. To illustrate the convergence property of the hen amplitude as a function of $\max \left(j_{i}\right)$, we show in Table II the results obtained for $\max \left(j_{i}\right)=3,4$ and 5. The table gives the real parts of individual contributions from the indicated types of the transition operators, calculated with the INOY interaction for $\Lambda=700 \mathrm{MeV}$. One can see that, with $\max \left(j_{i}\right)=5$ (used in the present work), the numerical accuracy of a few percent or better is achieved. The leading one-body (1B:LO) $\# 7$ contribution shows the slowest convergence, which can be understood by recalling that the $1 \mathrm{~B}$ impulse contribution undergoes a huge cancelation due to the orthogonality between the incoming and outgoing

\#7 Among the one-body M1 operator given in eq.(15), the 1B:LO corresponds to

$$
\boldsymbol{\mu}_{1 \mathrm{~B}: \mathrm{LO}}(q)=\frac{1}{2 m} \sum_{i}\left[\mu_{i} \hat{j}_{0}\left(q r_{i}\right) \boldsymbol{\sigma}_{i}+Q_{i} \hat{j}_{1}\left(q r_{i}\right) \boldsymbol{r}_{i} \times \overline{\boldsymbol{p}}_{i}\right]
$$


TABLE II: The real parts of contributions to the hen amplitude from the indicated types of the transition operators calculated for three different values of $\max \left(j_{i}\right)$, the maximal value of partial angular momenta $j_{i}$ allowed in the expansion of FYAs. We show here the results obtained with the INOY wave functions and with $\Lambda=700 \mathrm{MeV}$. The one-body leading order (1B:LO) contribution represents the impulse approximation terms, while 1B:RC corresponds to the relativistic corrections to the one-body current. The finite-range two-body current is decomposed into NLO one-pion exchange $(1 \pi), \mathrm{N}^{3} \mathrm{LO}$ pion-loop corrections $(1 \pi C)$ and $\mathrm{N}^{3} \mathrm{LO}$ two-pion exchange $(2 \pi)$ terms. The contact-type two-body current is decomposed into the $g_{4 s}$ and $g_{4 v}$ terms. All the matrix elements are given in units of $\mathrm{fm}^{3 / 2}$. For the values of $g_{4 s}$ and $g_{4 v}$ relevant to the present case, see Table III.

\begin{tabular}{lrrr}
\hline \hline & $\max \left(j_{i}\right) \leq 3$ & $\max \left(j_{i}\right) \leq 4$ & $\max \left(j_{i}\right) \leq 5$ \\
\hline \hline 1B: LO & 0.0455 & 0.0496 & 0.0511 \\
1B: RC & 0.0537 & 0.0535 & 0.0534 \\
1B-total & 0.0992 & 0.1031 & 0.1045 \\
\hline 2B: $1 \pi(\mathrm{NLO})$ & -0.0771 & -0.0781 & -0.0786 \\
2B: $1 \pi C\left(\mathrm{~N}^{3} \mathrm{LO}\right)$ & -0.0855 & -0.0861 & -0.0866 \\
2B: $2 \pi\left(\mathrm{N}^{3} \mathrm{LO}\right)$ & -0.0380 & -0.0383 & -0.0384 \\
\hline finite $($ total $/ \mathrm{o} \mathrm{CT})$ & -0.2006 & -0.2025 & -0.2035 \\
\hline 2B: $g_{4 s}$ & $0.0471 g_{4 s}$ & $0.0472 g_{4 s}$ & $0.0473 g_{4 s}$ \\
$2 \mathrm{~B}: g_{4 v}$ & $-0.0718 g_{4 v}$ & $-0.0722 g_{4 v}$ & $-0.0725 g_{4 v}$ \\
$2 B: \mathrm{CT}\left(\mathrm{N}^{3} \mathrm{LO}\right)$ & -0.2473 & -0.2495 & -0.2507 \\
\hline Total & -0.1482 & -0.1464 & -0.1462 \\
\hline \hline
\end{tabular}

nuclear wave functions. We have also checked that the convergence pattern is similar for the results with other nuclear potentials as well as for the imaginary parts of the transition amplitudes.

It turns out that the $1 \mathrm{~B}: \mathrm{RC}$ is dominated by the last term of the 2 nd line of eq.(5)), which accounts more than $95 \%$ of $1 \mathrm{~B}: \mathrm{RC}$. Note that this term is proportional to $\omega$ (the energy of the emitted photon), and hence does not contribute to the magnetic-moment operators.

As can be seen from Table II, the calculated value of the hen cross section, $\sigma$, for AV18 (INOY) is too large (small) compared with the experimental value, $\sigma_{\text {exp }}$, while the results 
TABLE III: Real and imaginary parts of the hen matrix element $\mathcal{M}$ (in units of $\mathrm{fm}^{3 / 2}$ ) calculated for $\Lambda=700 \mathrm{MeV}$. Also listed are the values of $g_{4 s}$ and $g_{4 v}$ (in units of $\mathrm{fm}^{3}$ ) determined so as to reproduce the magnetic moments of the ${ }^{3} \mathrm{H}$ and ${ }^{3} \mathrm{He}$ nuclei. $\Re \mathcal{M}$ is written in the format of: (1B) $+(2 \mathrm{~B} \mathrm{w} / \mathrm{o} \mathrm{CT})+\left(g_{4 s}\right.$-term $)+\left(g_{4 v}\right.$-term $)=($ total $)$.

\begin{tabular}{crrcc}
\hline \hline & $g_{4 s}$ & $g_{4 v}$ & $\Re \mathcal{M}$ & $\Im \mathcal{M}$ \\
\hline AV18 & 0.3958 & 0.1947 & $0.1531-0.3777+0.0237 g_{4 s}-0.0403 g_{4 v}=-0.2231$ & 0.0249 \\
I-N3LO & 0.3919 & 2.7479 & $0.1304-0.2248+0.0198 g_{4 s}-0.0371 g_{4 v}=-0.1885$ & 0.0203 \\
INOY & 0.2313 & 0.8021 & $0.1045-0.2035+0.0473 g_{4 s}-0.0725 g_{4 v}=-0.1462$ & 0.0154 \\
AV18+UIX & 0.5810 & -0.4615 & $0.1518-0.3567+0.0205 g_{4 s}-0.0377 g_{4 v}=-0.1756$ & 0.0179 \\
I-N3LO+UIX* & 0.5402 & 2.3249 & $0.1305-0.2253+0.0175 g_{4 s}-0.0347 g_{4 v}=-0.1661$ & 0.0183 \\
\hline \hline
\end{tabular}

for the remaining three nuclear interactions exhibit only mild variations around $\sigma_{\text {exp }}$. A detailed discussion of this model dependence will be given in the next subsection, but this is a good place to discuss certain features specific to AV18 and INOY. First, the conspicuous deviation of $\sigma(\mathrm{AV} 18)$ from $\sigma_{\text {exp }}$ is not surprising, since AV18 without additional three-body nuclear interactions fails to reproduce the binding energies of the relevant nuclei. We also remark that, although the INOY potential is capable of reproducing the binding energies and rms radii of the $A=3$ system quite accurately [43], it is known to give too large binding energies and too small rms radii for $A \geq 4$ nuclei [44] ; it also gives overbound and too dense nuclear matter [45]. Such a feature leads to the reduction of the overlap between the $n-{ }^{3} \mathrm{He}$ and ${ }^{4} \mathrm{He}$ wave functions and hence to underestimation of $\sigma$. It is also to be noted that the results for INOY deviate from the Tjon-line (a line that correlates the $A=3$ and $A=4$ binding energies) rather severely, indicating that caution should be exercised in using INOY for the $A \geq 4$ systems.

For further discussion, we list in Table III the values of the hen matrix element $\mathcal{M}$ [eq.(44)], and the LECs, $g_{4 s}$ and $g_{4 v}$, evaluated at $\Lambda=700 \mathrm{MeV}$, for each of the five nuclear interactions under consideration. The real part of $\mathcal{M}$ is written as the sum of $1 \mathrm{~B}$ and $2 \mathrm{~B}$ contributions, with the dependence on $g_{4 s}$ and $g_{4 v}$ also shown. We see from the table that the $2 \mathrm{~B}$ contributions are about two times as large as the $1 \mathrm{~B}$ contributions and that the $2 \mathrm{~B}$ and 1B terms have opposite signs. These features are consistent with the observation made in [14, 16]. Secondly, there are substantial model dependence even in the 1B sector, which 
might be traced to the fact that not all the adopted nuclear potentials accurately reproduce the ERPs that govern the long-r contributions of 1B. Finally, the inclusion of TNI(UIX) plays quite an important role in reducing the model dependence.

\section{A. Model-dependence}

As mentioned, the calculated values of the hen cross section $\sigma$, shown in Table \exhibit significant dependence on the nuclear interactions used. In examining this model dependence, it is informative to recall the results of our previous MEEFT study [21] on the M1 properties of the $A \leq 3$ nuclei. It was found in [21] that the M1 matrix elements (MEs) of the $A=3$ systems and the triton binding energy $B_{3}$ calculated for various realistic nuclear interactions exhibit strong correlations and they lie on a well-defined curve in the MEs- $B_{3}$ plot. Meanwhile, since $B_{3}$ governs the long-distance contributions to the MEs, the modeldependence (viz., variations in the MEs corresponding to the different nuclear potentials that give different values for $B_{3}$ ) cannot be cured by renormalizing the local (or short-ranged) operators. As discussed in [21], however, the use of the empirical correlation curve between the MEs and $B_{3}$ allows us to drastically reduce scatter in the calculated values of the MEs. This is achieved by introducing a constraint that only those values of the MEs be accepted which, along the correlation curve, have values of $B_{3}$ consistent with its experimental value. This constraint was found to essentially eliminate the model dependence in the MEs [21].

We expect that in principle a similar procedure can be adopted for the hen process. To this end, it seems useful to find a quantity that is related to the ERS in the $A=4$ systems and that exhibits strong correlation with the hen cross section, $\sigma$. We define the quantity $\zeta$ by

$$
\zeta \equiv\left[q\left(a_{n \mathrm{He} 3} / r_{\mathrm{He} 4}\right)^{2}\right]^{-2.75}
$$

where $q=\mathrm{BE}\left({ }^{4} \mathrm{He}\right)-\mathrm{BE}\left({ }^{3} \mathrm{He}\right)$, and the other quantities have already appeared in Table I. Fig. 2 2 shows the calculated values of $\sigma$ as a function of $\zeta$. Strong linear dependence between $\sigma$ and $\zeta$ can be seen. Suppose we take this correlation seriously and consider the quantity $\tilde{\sigma}$ defined by

$$
\tilde{\sigma} \equiv \frac{\zeta_{\exp }}{\zeta} \sigma
$$




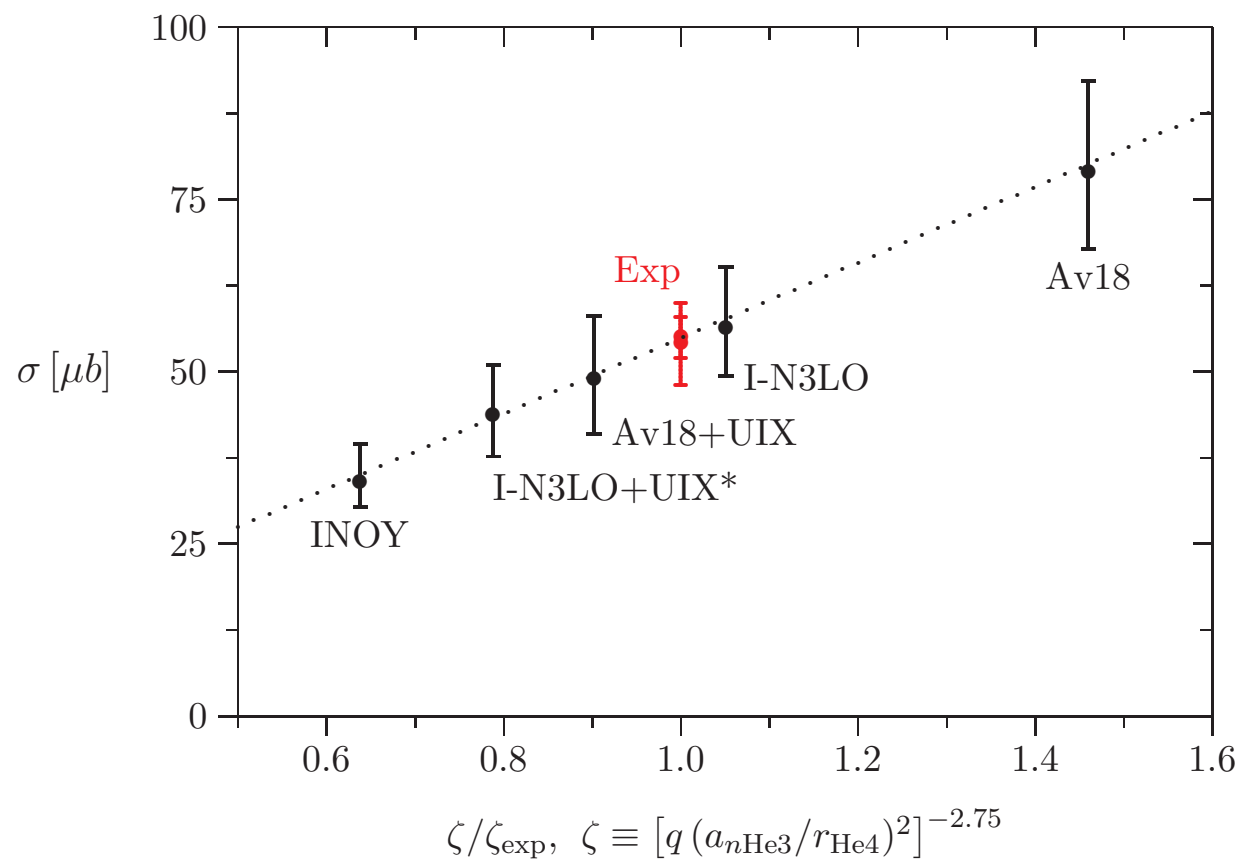

FIG. 2: (Color online) The hen cross section $\sigma$ (in units of $\mu b$ ) plotted against $\zeta / \zeta_{\exp }$, where $\zeta=\left[q\left(a_{n \mathrm{He} 3} / r_{\mathrm{He} 4}\right)^{2}\right]^{-2.75}$, and $\zeta_{\exp }$ is the value of $\zeta$ when all the quantities therein are given their respective empirical values (See Table I). For each indicated nuclear interaction, the error bar represents the range of variation for three different choices of the cutoff parameter: $\Lambda=500 \mathrm{MeV}$ (lower end), $700 \mathrm{MeV}$ (filled circle) and $900 \mathrm{MeV}$ (upper end). The lines in red with label "Exp" denote the experimental data, $54 \pm 6 \mu b[9]$ and $55 \pm 3 \mu b[10]$.

where $\zeta_{\exp }$ is the value of $\zeta$ when all the quantities in eq.(19) are given their respective empirical values. Then $\tilde{\sigma}$ turns out to be almost model-independent:

$$
\tilde{\sigma}=(54.8 \pm 8.4,54.5 \pm 7.5,53.9 \pm 7.1,54.8 \pm 9.4,56.4 \pm 8.5) \mu b
$$

for AV18, I-N3LO, INOY, AV18+UIX and I-N3LO+UIX*, respectively, and these values are all in agreement with data.

It is however not quite clear whether the correlation between $\sigma$ and $\zeta$ is accidental or physical in nature. Furthermore, since hen is a four-body process that involve a large number of ERPs to be controlled, our numerical results that only cover five different nuclear interactions may not be sufficient to establish the meaning of correlation unambiguously. For example, a correlation similar to the one between $\sigma-\zeta$ can be seen if we plot $\sigma$ against $q^{5} P_{D}^{2 / 3}$. (It is however not clear whether this correlation is independent of the $\sigma-\zeta$ correlation. They can be just different ways to express the same correlation, because $P_{D}$ and $r_{\mathrm{He} 4}$ may not be 
independent of each other.) Without delving into the discussion of physics behind the $\sigma-\zeta$ correlation, we take here the viewpoint that the $\sigma$ calculated with a nuclear interaction that does not reproduce the relevant ERPs should be considered much less reliable than the $\sigma$ obtained with a nuclear interaction that does reproduce the relevant ERPs. Based on this viewpoint, we adopt here the results obtained with AV18+UIX and I-N3LO+UIX* as the most reliable theoretical values for $\sigma$. For these two cases, the calculated hen cross sections are:

$$
\sigma=49.4 \pm 8.5 \mu b(\mathrm{AV} 18+\mathrm{UIX}), \quad \sigma=44.4 \pm 6.7 \mu b\left(\mathrm{I}-\mathrm{N} 3 \mathrm{LO}+\mathrm{UIX}^{*}\right)
$$

These values are in good agreement with the data within experimental errors.

\section{B. Comparison of our results with previous studies}

Making comparison of our calculation with the other related studies is not straightforward due to differences in the employed current operators and the nuclear wave functions; here we limit ourselves to discussion of the 1B:LO contribution. Let $\sigma_{1 \mathrm{~B}: \mathrm{LO}}$ be the hen cross section arising from the 1B:LO term. In our calculation, $\sigma_{1 \mathrm{~B}: \mathrm{LO}}$ varies from $4 \mu \mathrm{b}$ (for INOY) to $\sim 16$ $\mu \mathrm{b}$ (for AV18), which agrees well with the range of values $(2 \sim 14) \mu \mathrm{b}$ obtained by Towner and Khanna [11] using rather schematic wave functions.

It seems more significant to compare our results with those of Ref. [14], where the authors used the variational Monte-Carlo (VMC) method with the AV14+UVII potentials and obtained $\sigma_{1 \mathrm{~B}: \mathrm{LO}}=5.65 \mu \mathrm{b}$. Our calculation with AV18+UIX gives $\sigma_{1 \mathrm{~B}: \mathrm{LO}}=14.9 \mu \mathrm{b}$, which is about 2.6 times larger than the result of Ref. [14]. Since the 1B:LO M1 operator is the same for both cases, the discrepancy should be traced to the difference in the nuclear wave functions. Part of the discrepancy can be attributed to the fact that the calculated values of the ERPs vary for different realistic nuclear interactions used. For the $n-{ }^{3}$ He scattering length $a_{n \mathrm{He} 3}$, Ref.[14] reports $\Re\left(a_{n \mathrm{He} 3}\right)=3.5 \mathrm{fm}$ for AV14+UVII, and that $\sigma_{1 \mathrm{~B}: \mathrm{LO}}$ is increased by about $40 \%$ if $a_{n \mathrm{He} 3}$ is reduced from $3.5 \mathrm{fm}$ to $3.25 \mathrm{fm}$, which is close to $3.23 \mathrm{fm}$ for AV18+UIX. However this feature can explain only small part of the discrepancy.

An explanation for the remaining discrepancy may lie in the fact that the large- $r$ boundary condition for the initial scattering state adopted in Ref. [14] does not take into account coupling between the $n-{ }^{3} \mathrm{He}$ and the $p-{ }^{3} \mathrm{H}$ states. Such a simplification may be considered 
rather unwarranted for the following reasons. First, the presence of the diverging factor $\sqrt{p_{p} / p_{n}}$ in eq.(18) enhances the coupling in the asymptotic region. Secondly, ignoring the coupling between $n-{ }^{3} \mathrm{He}$ and $p-{ }^{3} \mathrm{H}$ states breaks orthogonality between the incoming and outgoing wave functions. Since, as mentioned, the 1B:LO contribution undergoes a huge suppression due to the pseudo-orthogonality, even a small breaking of the orthogonality can have very strong influence on the 1B:LO contribution. Finally, the variational calculation in [6] involves too few correlation operators to accurately describe the non S-wave components (the ones coupled by the IA operator) in the variational wave function.

It is noteworthy that a very similar feature occurs in hep calculations. That is, the correlated-hyperspherical harmonics method [49] with AV18+UIX leads to a 1B $S$-factor that is about four times larger than that obtained in the VMC calculation [6] with the AV14+UVIII; this additional example seems to render support to our above argument.

As mentioned in section I. after the submission of this work, there has appeared an elaborate calculation of hen [13], in which both the potential and current operators are derived from chiral effective field theory using time ordered perturbation theory. Ref. [13] gives $50 \pm 6 \mu b$ for the total hen cross section. Despite the differences in the details of the formulation, the general features of the calculation and the numerical results are in agreement between our work and Ref. [13].

\section{Cutoff dependence}

We now turn our attention to the cutoff dependence. Table IV shows to what extent the hen matrix element $\mathcal{M}$ calculated for the AV18+UIX wave functions changes when the cutoff parameter $\Lambda$ is varied over a range $\Lambda=500 \sim 900 \mathrm{MeV}$. Table IV indicates that the renormalization procedure of the LECs, $g_{4 s}$ and $g_{4 v}$, plays an essential role in reducing the cutoff-dependence. As a way of quantifying this feature, we define the quantity

$$
R \equiv \frac{\mathcal{M}_{\text {total }}\left(\Lambda_{2}\right)-\mathcal{M}_{\text {total }}\left(\Lambda_{1}\right)}{\mathcal{M}_{\text {finite }}\left(\Lambda_{2}\right)-\mathcal{M}_{\text {finite }}\left(\Lambda_{1}\right)}
$$

where the subscript "finite" stands for "finite-range term contributions", and $\mathcal{M}_{\text {finite }}$ corresponds to a case where all the terms other than the contact term $(\mathrm{CT})$ contributions are included. Perfect renormalization invariance would correspond to $R=0$. It turns out that $R_{\text {hen }}=0.189$ for $\left(\Lambda_{1}, \Lambda_{2}\right)=(500,900) \mathrm{MeV}$. Thus the renormalization procedure of LECs 
TABLE IV: Cutoff-dependence of the hen matrix element $\mathcal{M}$ calculated with the AV18+UIX wave functions. The hen cross section $\sigma$ is in units of $\mu b$; for other explanations, see the caption for Table III

\begin{tabular}{crrccc}
\hline \hline$\Lambda[\mathrm{MeV}]$ & $g_{4 s}$ & $g_{4 v}$ & $\Re \mathcal{M}$ & $\Im \mathcal{M}$ & $\sigma$ \\
\hline 500 & 0.8366 & 1.9068 & $-0.0915+0.0246 g_{4 s}-0.0471 g_{4 v}=-0.1608$ & 0.0166 & 40.9 \\
600 & 0.6990 & 0.5886 & $-0.1593+0.0231 g_{4 s}-0.0433 g_{4 v}=-0.1686$ & 0.0173 & 44.9 \\
700 & 0.5810 & -0.4615 & $-0.2049+0.0205 g_{4 s}-0.0377 g_{4 v}=-0.1756$ & 0.0179 & 48.7 \\
800 & 0.4517 & -1.3622 & $-0.2346+0.0176 g_{4 s}-0.0319 g_{4 v}=-0.1832$ & 0.0186 & 52.9 \\
900 & 0.3169 & -2.2069 & $-0.2547+0.0149 g_{4 s}-0.0265 g_{4 v}=-0.1915$ & 0.0195 & 57.9 \\
\hline \hline
\end{tabular}

has removed a major part of cutoff-dependence; the cutoff-dependence of $\mathcal{M}_{\text {total }}$ is about one-fifth of that of $\mathcal{M}_{\text {finite }}$. It is interesting to compare the above value of $R_{\text {hen }}$ with the corresponding quantity $R_{h e p}$ obtained in a hep calculation in [7]. The hep calculation in [7] is based on the same MEEFT strategy (but uses a different method for obtaining exact solutions to the nuclear Schröedinger equations). Ref. [7] reports $R_{h e p}=0.137$ for the slightly smaller window, $\left(\Lambda_{1}, \Lambda_{2}\right)=(500,800) \mathrm{MeV}$. Thus the previous hep calculation [7] is consistent with the hen calculation in the present work, and this consistency provides further support to the hep results in [7].

\section{Convergence of chiral expansion}

Table $\mathrm{V}$ shows the individual contributions of the various $1 \mathrm{~B}$ and $2 \mathrm{~B}$ terms to $\mu\left({ }^{3} \mathrm{H}\right)$, $\mu\left({ }^{3} \mathrm{He}\right)$ and the hen matrix element $\mathcal{M}$, calculated at $\Lambda=700 \mathrm{MeV}$ for the AV18+UIX potential. We can see that the $1 \mathrm{~B}$ contribution to hen is highly suppressed due to the aforementioned orthogonality between the initial and final wave functions. The NLO contribution, which comes from the soft one-pion-exchange, is also suppressed for the M1 channel, due to the accidental cancelation between the pion-pole and pion-seagull diagram contributions [21]. These suppression mechanisms make chiral convergence rather unclear. For example, one might worry about the fact that the $1 \pi C$ and $2 \pi$ contributions, both of which are $\mathrm{N}^{3} \mathrm{LO}$, turn out to be comparable in size to the NLO $1 \pi$ contribution. It should be noted, however, that most of the $1 \pi C$ and $2 \pi$ contributions are to be absorbed in the renormaliza- 
TABLE V: The magnetic moments, $\mu\left({ }^{3} \mathrm{H}\right)$ and $\mu\left({ }^{3} \mathrm{He}\right)$, and the real and imaginary parts of the hen matrix element, $\mathcal{M}$, calculated for the AV18+UIX wave functions and for $\Lambda=700 \mathrm{MeV}$. The values of $\mu\left({ }^{3} \mathrm{H}\right)$ and $\mu\left({ }^{3} \mathrm{He}\right)$ given in the row labeled "Total" are experimental values, which are used to fix the LECs. The LECs corresponding to this case are: $\left(g_{4 s}, g_{4 v}\right)=(0.581,-0.4615)\left[\mathrm{fm}^{3}\right]$.

\begin{tabular}{|c|c|c|c|c|}
\hline & $\mu\left({ }^{3} \mathrm{H}\right)$ & $\mu\left({ }^{3} \mathrm{He}\right)$ & $\Re \mathcal{M}$ & $\Im \mathcal{M}$ \\
\hline 1B: LO & 2.5727 & -1.7632 & 0.0964 & -0.0136 \\
\hline 1B: RC & -0.0171 & 0.0037 & 0.0554 & -0.0075 \\
\hline 1B-total & 2.5556 & -1.7595 & 0.1518 & -0.0211 \\
\hline $2 \mathrm{~B}: 1 \pi(\mathrm{NLO})$ & 0.2292 & -0.2258 & -0.1657 & 0.0195 \\
\hline $2 \mathrm{~B}: 1 \pi C\left(\mathrm{~N}^{3} \mathrm{LO}\right)$ & 0.1578 & -0.1289 & -0.1465 & 0.0172 \\
\hline $2 \mathrm{~B}: 2 \pi\left(\mathrm{N}^{3} \mathrm{LO}\right)$ & 0.0419 & -0.0408 & -0.0445 & 0.0052 \\
\hline \multirow[t]{3}{*}{ finite (total w/o CT) } & 2.9845 & -2.1550 & -0.2049 & 0.0208 \\
\hline & $0.0193 g_{4 s}$ & $0.0190 g_{4 s}$ & $0.0205 g_{4 s}$ & $-0.0014 g_{4 s}$ \\
\hline & $+0.0363 g_{4 v}$ & $-0.0354 g_{4 v}$ & $-0.0377 g_{4 v}$ & $+0.0044 g_{4 v}$ \\
\hline 2B: $\mathrm{CT}\left(\mathrm{N}^{3} \mathrm{LO}\right)$ & $=-0.0055$ & $=0.0274$ & $=0.0293$ & $=-0.0029$ \\
\hline Total & 2.9790 & -2.1276 & -0.1756 & 0.0179 \\
\hline
\end{tabular}

tion of the LECs, leaving very small net effects on the observable quantities. To demonstrate this point, we define the effective matrix element, $\langle\mathcal{O}\rangle^{\text {effective }}$, of a given operator $\mathcal{O}$ by

$$
\langle\mathcal{O}\rangle^{\text {effective }} \equiv \mathcal{M}_{\text {total }}-\left(\mathcal{M}_{\text {total }} \text { but without }\langle\mathcal{O}\rangle\right)
$$

Thus $\langle\mathcal{O}\rangle^{\text {effective }}$ represents a net change in the amplitude that would occur if we omit the operator $\mathcal{O}$. In evaluating the parenthesized quantity, the LECs should be readjusted so as to reproduce the experimental values of the $A=3$ magnetic moments without $\langle\mathcal{O}\rangle$; because of this readjustment we should expect $\langle\mathcal{O}\rangle^{\text {effective }} \neq\langle\mathcal{O}\rangle$. We find

$$
\begin{aligned}
\langle 1 \pi\rangle^{\text {effective }}=0.0749-0.0087 i, & \left(g_{4 s}, g_{4 v}\right)=(0.5223,5.8848) \mathrm{fm}^{3}, \\
\langle 1 \pi C\rangle^{\text {effective }}=-0.0093+0.0004 i, & \left(g_{4 s}, g_{4 v}\right)=(1.2433,3.53455) \mathrm{fm}^{3} \\
\langle 2 \pi\rangle^{\text {effective }}=-0.0010+0.0001 i, & \left(g_{4 s}, g_{4 v}\right)=(0.5843,0.6921) \mathrm{fm}^{3}
\end{aligned}
$$

where we have also listed the corresponding values of the LECs, which should be compared with $\left(g_{4 s}, g_{4 v}\right)=(0.581,-0.4615) \mathrm{fm}^{3}$ that correspond to the full calculation up to $\mathrm{N}^{3} \mathrm{LO}$. 
Eq.(25) demonstrates that the effective contributions of $1 \pi C$ and $2 \pi$ are very small, only about $6 \%$ and $2 \%$, respectively, relative to the values one would naively expect. This is in sharp contrast with the NLO soft one-pion-exchange, whose contribution cannot be absorbed in the LECs. A rigorous examination of chiral convergence would require a calculation that goes one order higher than considered in the present work (i.e., we need to go up to $\mathrm{N}^{4} \mathrm{LO}$ ), but we relegate this task to future studies.

There can also be a fully consistent EFT approach where both nuclear interactions and transition operators are obtained in the same EFT framework. This approach requires much more involved calculations than MEEFT, but recent significant progress in constructing EFT Hamiltonians makes it more attractive. Also available is a pionless EFT approach [47] where the matrix elements are evaluated perturbatively, but, unless it is capable of reproducing all the relevant ERPs of the nuclear systems involved, its usefulness is limited.

\section{DISCUSSION AND CONCLUSIONS}

In this work we have performed an $a b$ initio parameter-free calculation for the hen cross section $\sigma$, with the use of the EM currents that have been derived from HBChPT up to $\mathrm{N}^{3} \mathrm{LO}$. The exact nuclear wave functions for the initial and final states have been obtained by solving the Faddeev-Yakubovsky equations for realistic nuclear interactions. The calculated value of $\sigma$ shows a high degree of stability as the cutoff parameter $\Lambda$ is varied over a wide range, $\Lambda=(500 \sim 900) \mathrm{MeV}$, and we obtain as the best estimate $\sigma=49.4 \pm 8.5 \mu b$ for AV18+UIX and $44.4 \pm 6.7 \mu b$ for I-N3LO+UIX*. These values are in good agreement with the data, $54 \pm 6 \mu b[9]$ and $55 \pm 3 \mu b[10]$.

The successful application of MEEFT to hen renders strong support to the previous

MEEFT calculation of hep in Ref. [7]; furthermore, it demonstrates the basic soundness of the MEEFT approach in general. The present treatment is open to several improvements such as: the inclusion of the next order terms in chiral perturbation, in particular the incorporation of the three-nucleon currents; a more stringent control of mismatch in the chiral counting between SNPA and a formally accurate chiral expansion that enters in the currents; a better understanding of the role the counter terms play in the renormalization group property. It is reasonable, however, to expect that the effects of these improvements are essentially accommodated in the above-quoted error estimate based on the cutoff de- 
pendence. A robust estimation of the hep S-factor has been a long-standing challenge in nuclear physics [46]. We believe that our MEEFT calculations of hep and hen have solved this problem to a satisfactory degree.

\section{Acknowledgement}

The work of TSP is supported by the Korea Science and Engineering Foundation (KOSEF) Basic Research Program with the grant No. R01-2006-10912-0, by the KOSEF grant funded by the Korea Government (MEST) (No. M20608520001-08B0852-00110) and by the US National Science Foundation, Grant No. PHY-0758114.

YHS would like to express his gratitude to Professor Dong-Pil Min who encouraged him to start this work. The work of YHS was partly supported by the Korea Research Foundation Grant funded by the Korean Government (MOEHRD, Basic Research Promotion Fund) (KRF-2008-357-C00021) and the US Department of Energy under Contract No. DE-FG0209ER41621.

We are deeply obliged to Professors Mannque Rho and Kuniharu Kubodera for kindly agreeing to read the manuscript prior to its publication and giving us important comments.

The numerical calculations have been performed at IDRIS (CNRS, France). We thank the staff members of the IDRIS computer center for their constant help.

[1] K. Kubodera and T.-S. Park, Ann. Rev. Nucl. Part. Sci. 54 (2004) 19.

[2] INT workshop on "Solar Fusion Cross Sections for the pp chain and CNO cycle", Institute for Nuclear Theory, Seattle, USA, January 21-23, 2009.

[3] J. Carlson, D.O. Riska, R. Schiavilla and R.B. Wiringa Phys. Rev. C 44 (1991) 619.

[4] L.E. Marcucci, R. Schiavilla, M. Viviani, A. Kievsky, and S. Rosati, Phys. Rev. Lett. 84 (2000) 5959 .

[5] L.E. Marcucci, R. Schiavilla, M. Viviani, A. Kievsky, S. Rosati, J.F. Beacom, Phys. Rev. C 63 (2000) 015801.

[6] R. Schiavilla, R.B. Wiringa, V.R. Pandharipande and J. Carlson, Phys. Rev. C 45 (1992) 2628. 
[7] T.-S. Park, L.E. Marcucci, R. Schiavilla, M. Viviani, A. Kievsky, S. Rosati, K. Kubodera, D.-P. Min and M. Rho, Phys. Rev. C 67 (2003) 055206.

[8] G.E. Brown and M. Rho, Phys. Rept. 396 (2004) 1.

[9] F.L.H. Wolfs, S.J. Freedman, J.E. Nelson, M.S. Dewey and G.L. Greene, Phys. Rev. Lett. 63 (1989) 2721.

[10] R. Wervelman, K. Abrahams, H. Postma, J.G.L. Booten and A.G.M. Van Hees, Nucl. Phys. A526 (1991) 265.

[11] I.S. Towner and F.C. Khanna, Nucl. Phys. A356 (1981) 445.

[12] L. Girlanda, S. Pastore, R. Schiavilla and M. Viviani, arXiv:0912.2753 [nucl-th].

[13] L. Girlanda, A. Kievsky, L. E. Marcucci, S. Pastore, R. Schiavilla and M. Viviani, Phys. Rev. Lett. 105, 232502 (2010).

[14] J. Carlson, D.O. Riska, R. Schiavilla and R.B. Wiringa, Phys. Rev. C 42 (1990) 830.

[15] M. Chemtob and M. Rho, Nucl. Phys. A163 (1971) 1.

[16] Y.-H. Song and T.-S. Park, arXiv:nucl-th/0311055.

[17] S. Pastore, R. Schiavilla and J. L. Goity, Phys. Rev. C78 (2008) 064002

[18] S. Kolling, E. Epelbaum, H. Krebs and U. G. Meissner, Phys. Rev. C 80, 045502 (2009) arXiv:0907.3437 [nucl-th]].

[19] S. Pastore, L. Girlanda, R. Schiavilla, M. Viviani and R. B. Wiringa, Phys. Rev. C 80, 034004 (2009) arXiv:0906.1800 [nucl-th]].

[20] Y.-H. Song, R. Lazauskas and T.-S. Park, Phys. Lett. B 656 (2007) 174.

[21] Y.-H. Song, R. Lazauskas and T.-S. Park, Phys. Rev. C79 (2009) 064002.

[22] T.-S. Park, D.-P. Min and M. Rho, Phys. Rev. Lett. 74 (1995)4153-4156; Nucl. Phys. A 596 (1996) 515; T.-S. Park, K. Kubodera, D.-P. Min and M. Rho, Phys. Rev. C 58 (1998) R637.

[23] T.-S. Park, K. Kubodera, D.-P. Min and M. Rho, Phys. Lett. B 472 (2000) 232.

[24] L.D. Faddeev, Zh. Eksp. Teor. Fiz. 39 (1960) 1459 (Wiley \& Sons Inc., 1972) [Sov. Phys. JETP 12 (1961) 1014].

[25] O.A. Yakubowsky, Sov. J. Nucl. Phys. 5 (1967) 937.

[26] R. Lazauskas, Ph.D. Thesis, Université Joseph Fourier, Grenoble (2003); http://tel.ccsd.cnrs.fr/documents/archives0/00/00/41/78/.

[27] R. Lazauskas, Few Body Syst. 46, 37 (2009) [arXiv:0808.1650 [nucl-th]].

[28] R.B. Wiringa, V.G.J. Stoks, R. Schiavilla, Phys. Rev. C 51 (1995) 38. 
[29] D.R. Entem and R. Machleidt, Phys. Rev. C 68 (2003) 041001(R).

[30] P. Doleschall, I. Borbély, Z. Papp, W. Plessas, Phys. Rev. C 67 (2003) 064005.

[31] B.S. Pudliner, V.R. Pandharipande, J. Carlson and R.B. Wiringa, Phys. Rev. Lett. 74 (1995) 4396.

[32] E. Caurier and P. Navrátil, Phys. Rev. C 73 (2006) 021302(R).

[33] I. Sick, Phys. Rev. C 77 (2008) 041302(R).

[34] A. Deltuva and A.C. Fonseca, Phys. Rev. C 75 (2007) 014005.

[35] A. Kievsky, S. Rosati, M. Viviani, L.E. Marcucci and L. Girlanda, J. Phys. G 35 (2008) 063101.

[36] A. Nogga, H. Kamada and W. Glöckle, Phys. Rev. Lett. 85 (2000) 944.

[37] A. Deltuva, private communication.

[38] H.M. Hofmann and G.M. Hale, arXiv:nucl-th/0211008.

[39] M.G. Huber, M. Arif, T.C. Black, W.C. Chen, T.R. Gentile, D.S. Hussey, D.A. Pushin, F.E. Wietfeldt and L. Yang, Phys. Rev. Lett. 102 (2009) 200401.

[40] P.R. Huffman, D.L. Jacobson, K. Schoen, M. Arif, T.C. Black, W.M. Snow and S.A. Werner, Phys. Rev. C 70 (2004) 014004.

[41] W. Ketter, W. Heil, G. Badurek, M. Baron, E. Jericha, R. Loidl and H. Rauch, Eur. Phys. J. A 27 (2006) 243.

[42] A. Arriaga, V.R. Pandharipande and R. Schiavilla, Phys. Rev. C 43 (1991) 983.

[43] R. Lazauskas and J. Carbonell, Phys. Rev. C 70 (2004) 044002.

[44] C. Forssen, E. Caurier and P. Navratil, Phys. Rev. C 79 (2009) 021303(R).

[45] M. Baldo and C. Maieron, Phys. Rev. C 72 (2005) 034005.

[46] J.N. Bahcall, M.H. Pinsonneault and S. Basu, Astrophys. J. 555 (2001) 990.

[47] P.F. Bedaque and U. van Kolck, Ann. Rev. Nucl. Part. Sci. 52 (2002) 339 and references therein.

[48] A. Nogga, H. Kamada, W. Glöckle and B.R. Barett, Phys. Rev. C 65 (2002) 054003.

[49] L. E. Marcucci, R. Schiavilla, M. Viviani, A. Kievsky and S. Rosati, Phys. Rev. Lett. 84 (2000) 5959. 


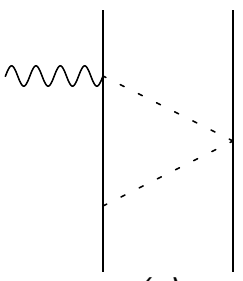

(a)

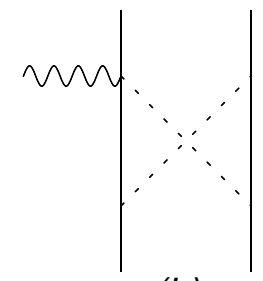

(b)

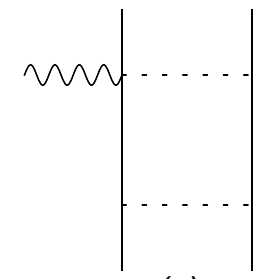

(c) 